\title{
COMMENTARY
}

\section{MicroRNA-21 regulates stemness in cancer cells}

\author{
Hong-Yo Kang \\ See related research by Chung et al., http://stemcellres.com/content/4/4/88
}

\begin{abstract}
MicroRNA-21 (miR-21) functions have been linked to cancer progression and chemo- or radiotherapy resistance. While an increasing number of studies have reported a potential role of miR-21 expression in promoting growth of a small population of stem/ progenitor cells, knowledge on its role as a regulator of stemness in cancers remains limited. In a previous issue of Stem Cell Research \& Therapy, Chung and colleagues provide evidence that miR-21 is highly expressed in stem/progenitor populations of ovarian teratocarcinoma cells and has the potential to mediate growth and self-renewal in cancer stem/progenitor cells. Here we summarize current knowledge on miR21 functions in human cancers and discuss how this finding provides insight into the role of miR-21 as an oncogenic regulator in stem/progenitor cell populations of human cancers.
\end{abstract}

More than 1,000 microRNAs (miRNAs/miRs) are expressed in human cells, where they participate in either pathological or physiological processes, affecting cell behavior and function and organismic phenotype. Among these, miR-21 has emerged as one of the most commonly and strongly upregulated miRs in a wide variety of human cancers. However, our understanding of the mechanisms of miR-21 elevation as a common feature in cancer remains rudimentary. The study by Chung and colleagues in a previous issue of Stem Cell Research \& Therapy discovered that miR-21 promotes ovarian teratocarcinoma cell growth through sustaining cancer stem/progenitor populations [1]. Their study provides new fundamental insights into the role of miR-21 as an oncogenic regulator in stem/progenitor cell

Correspondence: hkang3@mail.cgu.edu.tw Graduate Institute of Clinical Medical Sciences, Hormone Research Center, Kaohsiung Chang Gung Memorial Hospital, Chang Gung University College of Medicine, Kaohsiung City, Taiwan

Full list of author information is available at the end of the article populations that is involved in the promotion of the cellular transformation process and chemotherapy resistance.

miR-21 elevation has been demonstrated in miscellaneous human cancers, including glioblastoma, liver, gastric, cervical, thyroid, breast, prostate, colorectal, lung, pancreas, skin, and various lymphatic and hematopoietic cancers [2]. Although it has been identified as the best hit in a number of medium-scale and high-scale profiling experiments designed for the detection of miRNAs dysregulated in multiple cancer types, whether miR-21 exerts its oncogenic function on stem/progenitor populations of most types of cancers remains to be further investigated. One important point derived from this publication is that the increase in miR-21 expression in cancers may come from genetic changes underlying cancer stem/progenitor populations that lead to cancer progression, and chemo- or radiotherapy resistance. In a recent review on the oncogenic function of miR-21, this concept is further supported by evidence of altered promoter methylation of miR-21 associated with gene mutations in clear cell renal cell carcinoma [3] and that overexpression of miR-21 in mice leads to pre-B lymphoma formation [4]. Recent studies also reported that miR-21 could promote the migration and invasion of a stem-like population in hepatocellular carcinoma $[5,6]$. As it becomes progressively more evident that miR-21 may enhance cancer stem/progenitor cell growth, it would be of interest to elucidate by which mechanism miR-21 influences progenitor cells.

There are several possible mechanisms by which miR21 may promote cancer stem/progenitor populations: first, miR-21 in non-progenitor cancer cells could produce growth factors that enrich stem cell populations; second, miR-21 in the cancer progenitor cell niche might directly regulate progenitor cells to self-renew; third, miR-21 in certain non-progenitor cancer cells may trigger a dedifferentiation process, so enriching stem cell populations. Although a recent report showed that miR21 expression and function are associated with chemotherapy resistance, accompanied by increasing cancer stem/progenitor populations [7], as well as enriched side 
population cells (stem/progenitor cells) in hepatocellular carcinoma cell lines [6], there has been no clear dissection of the function of miR-21 in stem or non-stem populations of cancer cells.

In the report by Chung and colleagues, it was shown that miR-21 could promote the growth of ovarian teratocarcinoma PA1 cells, while knockdown of miR-21 could abolish cell growth. Furthermore, by dissecting CD133+ and CD133- cancer progenitor populations, they found that miR-21-mediated self-renewal of stem/progeny cells preferentially occurred in CD133+ cells. Therefore, the data presented in the article from Chung and colleagues favors the second hypothetical mechanism - that is, that miR-21 directly impacts on the progenitor cell population to promote cancer cell growth.

During homeostasis, miR-21 has been linked to cell growth and has emerged as one of the principal regulators controlling major cell functions. High levels of miR-21 may not only be a characteristic in cancer cells but also represent a common feature of pathological cell growth. For example, miR-21 is found to be essential for rapid growth of hepatic cells during liver regeneration [8]. Transient miR-21 expression after partial hepatectomy could suppress Rhob, subsequently relieving Akt/mTOR ablating effects on eIF/4F to trigger cyclin D1 translation and thus activating the cell cycle of mouse liver cells [8]. Interestingly, miR-21 is also upregulated in several models of mouse cardiac hypertrophy and in a variety of other human proliferative disorders [9], implying a function in regulating cell growth. This idea is further supported by evidence of miR-21 induction associated with maintaining mouse spermatogonial germ cell populations [10].

The accumulating data support an appealing concept that sequence-specific inhibition of miRNAs in stem/ progenitor cell populations can provide a novel therapeutic approach for modulation of stem/progenitor cells whose function is deregulated in cancer. In the study by Chung and colleagues, knockdown of miR-21 resulted in a marked reduction in the CD133+ population and sphere formation of stem/progenitor cells, thus inhibiting the growth of ovarian teratocarcinoma cells, suggesting such modulation has therapeutic potential. It is conceivable that modulation of miR-21 may sensitize stem/progenitor cells in modulating drug responses. It will be of great interest to investigate whether targeting miR-21 is one of the key approaches that enhance the susceptibility of cancer stem/progenitor cells to chemoand radiotherapeutic treatments. In conjunction with current therapeutic regimens, this may eventually lead to an effective strategy in the fight against these deadly cancers in the near future.

\section{Competing interests}

The author declares that they have no competing interests.

Published: 9 September 2013

\section{References}

1. Chung WM, Chang WC, Chen L, Chang YY, Shyr CR, Hung YC, Ma WL: MicroRNA-21 promotes the ovarian teratocarcinoma PA1 cell line by sustaining cancer stem/progenitor populations in vitro. Stem Cell Res Ther 2013, 4:88.

2. Krichevsky AM, Gabriely G: miR-21: a small multi-faceted RNA. J Cell Mol Med 2009, 13:39-53.

3. The Cancer Genome Atlas Research Network: Comprehensive molecular characterization of clear cell renal cell carcinoma. Nature 2013, 499:43-49.

4. Medina PP, Nolde M, Slack FJ: OncomiR addiction in an in vivo model of microRNA-21-induced pre-B-cell lymphoma. Nature 2010, 467:86-90.

5. Huang YH, Lin YH, Chi HC, Liao CH, Liao CJ, Wu SM, Chen CY, Tseng YH, Tsai CY, Lin SY, Hung YT, Wang CJ, Lin CD, Lin KH: Thyroid hormone regulation of miR-21 enhances migration and invasion of hepatoma. Cancer Res 2013, 73:2505-2517.

6. Zhou L, Yang ZX, Song WJ, Li QJ, Yang F, Wang DS, Zhang N, Dou KF: MicroRNA-21 regulates the migration and invasion of a stem-like population in hepatocellular carcinoma. Int J Oncol 2013, 43:661-669.

7. Yu Y, Sarkar FH, Majumdar AP: Down-regulation of miR-21 induces differentiation of chemoresistant colon cancer cells and enhances susceptibility to therapeutic regimens. Trans/ Oncol 2013, 6:180-186.

8. Ng R, Song G, Roll GR, Frandsen NM, Willenbring H: A microRNA-21 surge facilitates rapid cyclin D1 translation and cell cycle progression in mouse liver regeneration. J Clin Invest 2012, 122:1097-1108.

9. Thum T, Gross C, Fiedler J, Fischer T, Kissler S, Bussen M, Galuppo P, Just S, Rottbauer W, Frantz S, Castoldi M, Soutschek J, Koteliansky V, Rosenwald A, Basson MA, Licht JD, Pena JT, Rouhanifard SH, Muckenthaler MU, TuschI T, Martin GR, Bauersachs J, Engelhardt S: MicroRNA-21 contributes to myocardial disease by stimulating MAP kinase signalling in fibroblasts. Nature 2008, 456:980-984.

10. Niu Z, Goodyear SM, Rao S, Wu X, Tobias JW, Avarbock MR, Brinster RL: MicroRNA-21 regulates the self-renewal of mouse spermatogonial stem cells. Proc Natl Acad Sci U S A 2011, 108:12740-12745.

doi:10.1186/scrt321

Cite this article as: Kang: MicroRNA-21 regulates stemness in cancer cells. Stem Cell Research \& Therapy 2013 4:110. 